\title{
An efficient optimal solution method for the joint replenishment problem with minimum order quantities
}

\author{
Eric Porras ${ }^{*}$, Rommert Dekker \\ Tinbergen Institute, Econometric Institute, Erasmus University of Rotterdam, The Netherlands
}

\begin{abstract}
We study the joint replenishment problem (JRP) for $M$ items under deterministic demand, with a minimum order quantity constraint for each item in the replenishment order. We first study an iterative procedure that proves to be not efficient in this case. Further, we derive bounds on the basic cycle time and propose an efficient global optimisation procedure to solve the JRP with constraints. Moreover, we also consider the case where a correction is made for empty replenishment occasions. The algorithms are tested in a real case.
\end{abstract}

Keywords: Inventory; Joint replenishment; Minimum order quantities

\section{Introduction}

Minimum order quantities (MOQ) are often encountered in real supply chains, where companies enforce economies of scale due to high set-up costs associated with production or transportation processes. While costs can be associated to a total order consisting of several order lines for products, it is often difficult to determine set-up costs for individual order lines. This was observed in a real case [5]. In the methods presented so far for the deterministic joint replenishment problem (JRP) it is assumed that a major ordering cost is charged at a basic cycle time $T$ and that the ordering cycle of each item is some integer $k_{j}$ multiple of $T$, which is called a $\left(k_{j}, T\right)$ policy. Up to now, all research on the JRP has assumed existence of order line set-up costs and ignored minimal order quantities. In this line of research Goyal [4] and Van Eijs [2] proposed solution methods for the JRP based on enumeration of the frequencies $k_{j}$ 's. Viswanathan [6] and Fung and Ma [3] used tighter bounds on $T$ to improve the procedures by Goyal and Van Eijs. Wildeman, Frenk and Dekker [7] presented an efficient optimal solution method for large problems based on Lipschitz optimisation. We will adapt their formulation but develop a completely new method to make it suitable for minimum order quantities.

*Corresponding author: Tel.: +31-10-4082524

E-mail address: porrasmusalem@few.eur.nl 
In the next section we present the mathematical formulation of the JRP with MOQ. In section 3 we investigate an iterative method. Next we derive bounds on the basic cycle time and we include this information to develop a fast solution method. Contrary to many other papers, we explicitly deal with the correction factor for empty replenishments and we test the procedures using real data. The final conclusions are presented in the last section.

\section{The model}

We consider $M$ items that can be jointly replenished against a fixed cost $A$, independent of the quantity ordered. No minor set-up costs are associated with the order lines for the individual items, since a fixed size transportation cost is assumed, as in the case of container transportation. The demand is constant and known for the different items. We introduce the following notation:

$D_{j}: \quad$ constant rate of demand for item $j$

$h_{j}$ : $\quad$ unit holding cost per year for item $j$

$M: \quad$ number of items

$k_{j}: \quad$ replenishment frequency of item $j$ (decision variable)

k: $\quad$ vector of the $k_{j}$ 's, $j=1, \ldots, M$

$T: \quad$ basic cycle time (decision variable)

$M O Q_{j}:$ minimum order quantity for item $j$

Each $T$ time units there is a replenishment opportunity. Hence, every $k_{i} T$ time units item $i$ is replenished. Let $T C$ denote the average total cost of the system, which includes the ordering and the holding costs. We formulate the deterministic joint replenishment problem with minimum order quantities as follows:

$J R P$ with $M O Q$

$$
\min T C(T, \mathbf{k})=\frac{A}{T}+\frac{1}{2} T \sum_{j=1}^{M} h_{j} k_{j} D_{j}
$$

s.t. $\quad k_{j} D_{j} T \geq M O Q_{j} \quad$ for all $j$

$$
\begin{aligned}
& T>0 \\
& k_{j} \geq 1 \quad \quad \text { integers for } j=1,2, \ldots, M
\end{aligned}
$$

A standard way to solve problems with order size constraints is to introduce dummy order line set-up costs and manipulate them in such a way that the order restrictions are met. Note however that this requires multiple solving of the JRP, while the new method we will offer here is faster than solving the standard JRP. The formulation for the standard JRP is given below.

$J R P$ (standard formulation)

$$
\min T C^{(S)}(T, \mathbf{k})=\frac{A}{T}+\sum_{j=1}^{M}\left(\frac{a_{j}}{k_{j} T}+\frac{1}{2} h_{j} k_{j} D_{j} T\right)
$$


s.t. $\quad T>0$

$$
k_{j} \geq 1 \quad \text { integers for } j=1,2, \ldots, M
$$

where $a_{j}(j=1, \ldots, M)$ is the order line set-up cost of item $j$.

An alternative formulation of the function $T C$ includes the so-called correction factor $\Delta(\mathbf{k})$, which does not charge set up costs for empty replenishments, arising when the smallest $k_{j}$ is greater than one [1]. Accordingly we define the function $T C^{(c)}$ with the correction factor as follows:

$$
T C^{(c)}(T, \mathbf{k})=\frac{A \Delta(\mathbf{k})}{T}+\frac{1}{2} T \sum_{j=1}^{M} h_{j} k_{j} D_{j}
$$

where the factor $\Delta(\mathbf{k})$ is the fraction of non-empty replenishments per year.

This factor can be evaluated using the principle of inclusion-exclusion, counting the total fraction of replenishment occasions of all individual products, then subtracting (excluding) the joint replenishment fraction of all pairs of products, then adding (including) the joint replenishment fraction of all triples of products, and so on until all the joint fractions for the combined set of products have been included or excluded, as appropriate. Applying this principle the following formula is given for the evaluation of $\Delta(\mathbf{k})[1]$ :

$$
\begin{aligned}
& \Delta(\mathbf{k})=\sum_{i=1}^{M}(-1)^{i+1} \sum_{\{\alpha \subset\{1, \ldots, M\}:|\alpha|=i\}}\left(\operatorname{lcm}\left(k_{\alpha_{1}}, \ldots, k_{\alpha_{i}}\right)\right)^{-1} \\
& =\sum_{i=1}^{M} \frac{1}{k_{i}}-\sum_{(i, j) \subseteq\{1, \ldots, M\}} \frac{1}{\operatorname{lcm}\left(k_{i}, k_{j}\right)}+\sum_{(i, j, k) \subseteq\{1, \ldots, M\}} \frac{1}{\operatorname{lcm}\left(k_{i}, k_{j}, k_{k}\right)}-\cdots+(-1)^{M+1} \frac{1}{\operatorname{lcm}\left(k_{1}, \ldots, k_{M}\right)}
\end{aligned}
$$

where $\operatorname{lcm}\left(k_{\alpha_{1}}, \ldots, k_{\alpha_{i}}\right)$ denotes the least common multiple of the integers $k_{\alpha_{1}}, \ldots, k_{\alpha_{i}}$, i.e. the smallest number $n$ for which there exist positive integers $n_{\alpha_{1}}, \ldots, n_{\alpha_{i}}$ such that $n_{\alpha_{1}} k_{\alpha_{1}}=n_{\alpha_{2}} k_{\alpha_{2}}=\cdots=n_{\alpha_{i}} k_{\alpha_{i}}=n$. From the foregoing we can derive an important property that we will need later in our analysis.

Proposition 1. Given $M$ products with associated vector $\mathbf{k}$, the following holds:

$\sum_{i=1}^{M} \frac{1}{k_{i}}-\sum_{(i, j) \subseteq\{1, \ldots, M\}} \frac{1}{l c m\left(k_{i}, k_{j}\right)} \leq \Delta(\mathbf{k}) \leq \sum_{i=1}^{M} \frac{1}{k_{i}}$

Proof. As the fraction of replenishments of item $i$ per year is $\left(1 / k_{i}\right)$ it will be clear that the RHS holds. Now realise that through the principle of inclusion-exclusion the number of non-empty replenishments due to item $i$ is larger than the number of replenishments of item $i$ minus the joint replenishments of pairs of products including item $i$. Hence, the LHS of the inequality holds.

If at least one of the $k_{i}=1$, then $\Delta(\mathbf{k})=1$ and $T C^{(\mathrm{c})}(T, \mathbf{k})$ coincides with $T C(T, \mathbf{k})$. We will derive solution methods for the JRP with MOQ using both formulations for $T C$. 


\section{Solution methods for the JRP with MOQ}

For a fixed vector $\mathbf{k}=\left(k_{1}, \ldots, k_{M}\right)$, it is easy to verify that the function $T C$ is strictly convex in $T$. Thus, the $T$-value minimizing $T C$ for given $\mathbf{k}$ is

$$
T^{*}=\sqrt{\frac{2 A}{\sum_{j=1}^{M} h_{j} k_{j} D_{j}}}
$$

Taking into account the above result, we first investigate an iterative procedure inspired by the algorithm proposed by Viswanathan [6].

\subsection{Iterative algorithm to solve JRP with $M O Q$}

Step 0 . Set $l=0$. Set $k_{j}^{(0)}=1$ for $j=1, \ldots, M$.

Step 1. Fix $\mathbf{k}=\mathbf{k}^{(l)}$ and solve for $T^{*}$ using (4) and let $T^{(l)}=\max \left\{T^{*}, \max _{j}\left\{\frac{M O Q_{j}}{k_{j}^{(l)} D_{j}}\right\}\right\}$.

Step 2. Find the $k_{j}^{(l+1)}$ 's minimizing $T C\left(T^{(l)}, \mathbf{k}\right)$ using eqs. (1) and (3).

Inspection of the function $T C$ yields that the $k_{j}$ 's should be selected as small as possible satisfying (1). Hence,

$k_{j}^{(l+1)}=\left\lceil\frac{M O Q_{j}}{D_{j} T^{(l)}}\right\rceil$, where $\lceil x\rceil$ denotes the smallest integer greater or equal to $x$.

Evaluate $T C\left(T^{(l)}, \mathbf{k}^{(l+1)}\right)$.

If $\mathbf{k}^{(l+1)}=\mathbf{k}^{(l)}$ GOTO step 3. Else set $l=l+1$ and GOTO step 1.

Step 3. Evaluate $T C^{(c)}\left(T^{(l)}, \mathbf{k}^{(l+1)}\right)$, with corresponding correction factor $\Delta\left(\mathbf{k}^{(l+1)}\right)$.

The above algorithm has the possibility of getting trapped in the initial set of values for the $k_{j}$ 's. Nevertheless, it may well be that $M O Q_{j} \gg D_{j} T$ for most of the $j$. Therefore, due to the restriction on the MOQ's and assuming that differences in size do exist for demands across different items, the algorithm forces the values of $k_{j}$ to be different from 1, as we will show in the following numerical application.

Note that the iteration is defined only on $T C$ and not on $T C^{(c)}$. This is because an iteration on $T C^{(c)}$ does not need to converge as $T C^{(c)}$ is much more sensitive to $T$ than $T C$. Nevertheless, in step 3 of the algorithm we include the possibility of correcting the value of $T C$. 


\subsection{Numerical example based on real case}

We test the proposed procedures using data from a real supply chain for homogeneous products (gift items) transported by container from a manufacturer in China to a distribution centre in the Netherlands [5]. A staircase transport cost structure was identified, resulting from the use of container transportation. We make a simplification of the set-up cost and consider it constant, as if the company would always send a full container of items. As in that case the MOQ was applied to components (chips) of the final product, so we made groups of virtual products when they had the same chip component.

We apply the iterative algorithm to the following data:

$M=8$ items

$A=950$ euros per shipment (of a full container from China to the Netherlands)

$h_{j}=0.325$ euros/unit.year for all $j$

$M O Q_{j}=10,000$ units for all $j$

Table 1 shows the demand rates for the items.

\begin{tabular}{cc}
\multicolumn{2}{c}{ Table 1. Demand data } \\
\hline Item $j$ & Demand per week, $D_{j}$ \\
\hline 1 & 352 \\
2 & 388 \\
3 & 323 \\
4 & 195 \\
5 & 408 \\
6 & 195 \\
7 & 489 \\
8 & 489 \\
\hline
\end{tabular}

Given $\mathbf{k}^{(0)}=\{1,1, \ldots, 1\}$ and the above data step (1) of the algorithm gives: $T^{(0)}=0.199$ years $\approx 10.3$ weeks.

Step (2) now yields: $\mathbf{k}^{(1)}=\{3,3,4,5,3,5,2,2\}$ with associated annual average total cost $T C\left(T^{(0)}, \mathbf{k}^{(1)}\right)=19261$ euros/year.

Following step (1) with the above result for $\mathbf{k}$, we evaluate the new value of $T$ for the next iteration, resulting in: $T=\max \{0.114,0.197\}$ years $\approx 10.3$ weeks, and the algorithm stops in step (2). With the final solution for the $k_{j}$ 's, 4 out of 15 times the set-up cost will not be charged, i.e., a correction factor of $1-(4 / 15)$ should be applied to the total cost, so step (3) yields: $T C^{(c)}\left(T^{(1)}, \mathbf{k}^{(1)}\right)=17982$ euros/year.

We will now derive some bounds on $T$, with the idea of formulating a global optimisation procedure, thus avoiding the possibility of getting trapped in a local minimum of the function $T C$.

\subsection{Bounds on $T$}

Following the procedure of Wildeman et al [7], we can rewrite the original function $T C(T, \mathbf{k})$ as follows: 


$$
T C(T)=\frac{A}{T}+\sum_{j=1}^{M} z_{j}(T)
$$

where the function $z_{j}(T)$ is given by:

$z_{j}(T)=\min _{k_{j}}\left\{\frac{1}{2} h_{j} D_{j} k_{j} T \mid D_{j} k_{j} T \geq M O Q_{j} \forall j\right\}$.

A lower envelope $T C_{\text {low }}(T)$ for the function $T C(T)$ is found by setting $k_{j}=1$ for $j=$ $1, \ldots, M$, and removing the MOQ constraints. In Fig. 1 we show a plot of the function $T C(T)$ with its envelope using data from the numerical example, and a graphical procedure to find lower and upper bounds.

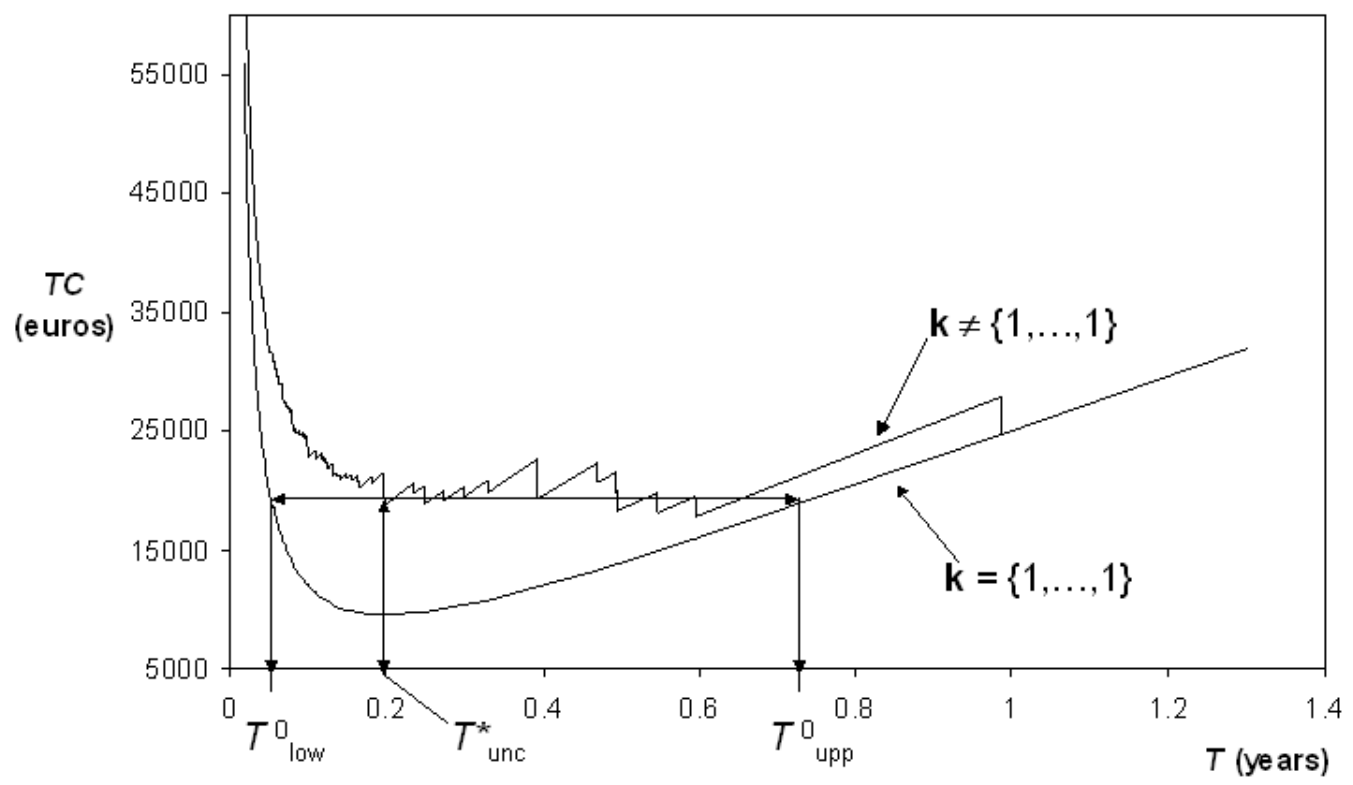

Figure 1. Plot of $T C(T)$ (upper curve) with its envelope $T C_{\text {low }}(T)$ (lower curve)

The envelope $T C_{\text {low }}(T)$ has an optimal unconstrained solution given by:

$$
T_{u n c}^{*}=\sqrt{\frac{2 A}{\sum_{j=1}^{M} h_{j} D_{j}}}
$$

For the constrained problem, the $k_{j}$ 's have to satisfy:

$k_{j} D_{j} T_{u n c}^{*} \geq M O Q_{j} \quad \forall j \quad \Rightarrow \quad k_{j}^{(0)}=\left\lceil\frac{M O Q_{j}}{D_{j} T_{u n c}^{*}}\right\rceil \quad$ for $\quad j=1, \ldots, M$

This yields a feasible solution for the JRP with MOQ. 
Taking into account the above result we can find a lower bound for $T C(T, \mathbf{k})$ by solving: $T C_{\text {low }}(T)=T C\left(T_{u n c}^{*}, \mathbf{k}^{(0)}\right)$, where $T C_{\text {low }}(T)$ is the unconstrained cost curve with $k_{j}=1$ for $j=1, \ldots, M$, which comes down to solving $T$ from the following quadratic equation:

$$
\frac{A}{T}+\frac{1}{2} T \sum_{j} h_{j} D_{j}=\frac{A}{T_{u n c}^{*}}+\frac{1}{2} T_{u n c}^{*} \sum_{j} h_{j}\left\lceil\frac{M O Q_{j}}{D_{j} T_{u n c}^{*}}\right\rceil D_{j}
$$

Let us call the resulting lower bound $T_{\text {low }}^{0}$ and the upper bound $T_{u p p}^{0}$. An alternative upper bound for $T$ can be found by setting $k_{j}=1$ for all $j$. Using the bounds for $T$, we can formulate a finite optimisation algorithm to solve the JRP with MOQ.

\subsection{Motivation for the optimisation algorithm}

Note that discontinuities of the function $T C(T)$ arise from the integrality of the $k_{j}$ 's and the requirements set by the minimum order quantities. At this point, we note that the vector $\mathbf{k}$ is a function of $T$, with its elements given by:

$$
k_{j}(T)=\left\lceil\frac{M O Q_{j}}{D_{j} T}\right\rceil \quad \forall j
$$

From (5) it follows that as $T$ decreases, a jump of the function $T C(T)$ will occur when at least one element of $\mathbf{k}$, say $k_{j}$, changes from $k_{j}$ to $k_{j}+1$. Note that the new vector $\mathbf{k}$ will remain constant until the next element of $\mathbf{k}$ changes in one unit. In other words, $T C(T)$ is piecewise convex in $T$ for the intervals in which the associated vectors k's remain unchanged. This important feature of the function $T C(T)$ allows us to make a partition of the set $T$ as follows:

Define $I_{i}$ as the interval $\left[T^{(i)}, T^{(i-1)}\right)$ inside which the function $T C(T)$ has an associated constant vector $\mathbf{k}^{(i-1)}$, with its elements given by (5). Now observe that for $T \in\left[T^{(i)}, T^{(i-1)}\right)$ the ratios $M O Q_{j} / D_{j} T$ increase as $T \rightarrow T^{(i)}$. The vector $\mathbf{k}^{(i-1)}$ will change when one (or more) of its elements increases by one unit just below $T^{(i)}$. Therefore, $T^{(i)}$ can be calculated from:

$$
T^{(i)}=\max _{j}\left\{\frac{M O Q_{j}}{D_{j} k_{j}^{(i-1)}}\right\}
$$

Then, the elements of the vector $\mathbf{k}$ just below $T^{(i)}$, say $\mathbf{k}^{(i)}$, are given by:

$$
k_{j}^{(i)}=\left\{\begin{array}{ccc}
k_{j}^{(i-1)}+1 & \text { for } & j \in J^{(i)} \\
k_{j}^{(i-1)} & \text { for } & j \notin J^{(i)}
\end{array}\right.
$$

where $J^{(i)}$ is the set of all coordinates of $\mathbf{k}$ for which the maximum in (6) is attained. 
To illustrate the previous ideas we present next a numerical example (see Fig. 2).

Let $\left\{\frac{M O Q_{j}}{D_{j}}\right\}_{j=1,2,3}=\{4,3,9\}$

Select an initial value of $T$, e.g. $T^{(0)}=0.85$ and evaluate the corresponding vector $\mathbf{k}^{(0)}$ using (5), to get $\mathbf{k}^{(0)}=\{5,4,11\}$. Note that the elements of the vector $\mathbf{k}^{(0)}$ will remain unchanged for $T$ inside the intervals:

$\frac{a_{j}}{k_{j}^{(0)}} \leq T<\frac{a_{j}}{k_{j}^{(0)}-1}$ for $j=1,2,3$ : (for $k_{j}=1$ the interval is given by: $a_{j} \leq T<\infty$ )
$0.8 \leq T<1$
$\left(k_{1}^{(0)}\right.$ remains constant)
$0.75 \leq T<1$
$\left(k_{2}^{(0)}\right.$ remains constant)
$0.818 \leq T<0.9$
$\left(k_{3}^{(0)}\right.$ remains constant)

Note that the vector $\mathbf{k}^{(0)}$ will remain constant for $T$ inside the interval $0.818 \leq T<0.9$. From this it is clear that as $T$ decreases, the first element of $\mathbf{k}^{(0)}$ to change is the one with largest $a_{j} / k_{j}$ ratio, $k_{3}^{(0)}$ in this case. In other words, the vector $\mathbf{k}(T)$ remains unchanged until $k_{3}(T)$ changes from 11 to 12 , just before $T^{(1)}=0.818$, and the new vector $\mathbf{k}^{(1)}=\{5,4,12\}$ is generated. At this point reset the value of $T^{(0)}$ to 0.9 , so the interval for which $\mathbf{k}^{(0)}$ remains constant is $T^{(1)} \leq T<T^{(0)}$.

Working backwards in time starting from $T^{(1)}$, we use (6) to get $T^{(2)}=0.8$, which is the time defining the next interval inside which $\mathbf{k}^{(1)}$ remains constant. So just before $T^{(2)}$ the new vector $\mathbf{k}^{(2)}$ is obtained by increasing in one unit the coordinate of $\mathbf{k}^{(1)}$ for which the maximum in (6) was attained, i.e., $\mathbf{k}^{(2)}=\{6,4,12\}$. Continuing like this, we get $T^{(3)}=0.75$, where the maximum in (6) was attained for coordinates 2 and 3 . Note that $\left[T^{(3)}, T^{(2)}\right)$ is the interval inside which $\mathbf{k}^{(2)}$ remains constant, and just before $T^{(3)}$ the new vector $\mathbf{k}^{(3)}=\{6,5,13\}$ is obtained.

Note that starting with the vector $\mathbf{k}^{(0)}=\{5,4,11\}$ there is no interval for $T$ inside which the vector $\{6,4,11\}$ could be produced, since the following sequence was obtained: $\{5,4,11\} \Rightarrow\{5,4,12\} \Rightarrow\{6,4,12\} \Rightarrow\{6,5,13\}$. In other words, there is a one-to-one correspondence between the intervals $I_{i}$ and the vectors $\mathbf{k}$.

The previous analysis suggests that we can search for the local minima of the function $T C(T)$ in each interval $I_{i}$ in the classical way, and then to compute the global minimum of the function among all intervals. From this it is clear that the problem $\min T C(T)$ s.t. $T>0$ is equivalent to:

$$
\min _{i} \min _{T \in I_{i}} T C_{\mathbf{k}^{(i-1)}}(T)
$$

We can start our search procedure in $T=T_{u p p}^{0}$, with associated vector $\mathbf{k}\left(T_{u p p}^{0}\right)$, and then find the time $T^{(1)}<T_{u p p}^{0}$ where the previous jump of the function took place. Continue like this working backwards in time stopping when the lower bound on $T$ is reached. 
In Fig. 2 a graphical representation of the algorithm is shown.

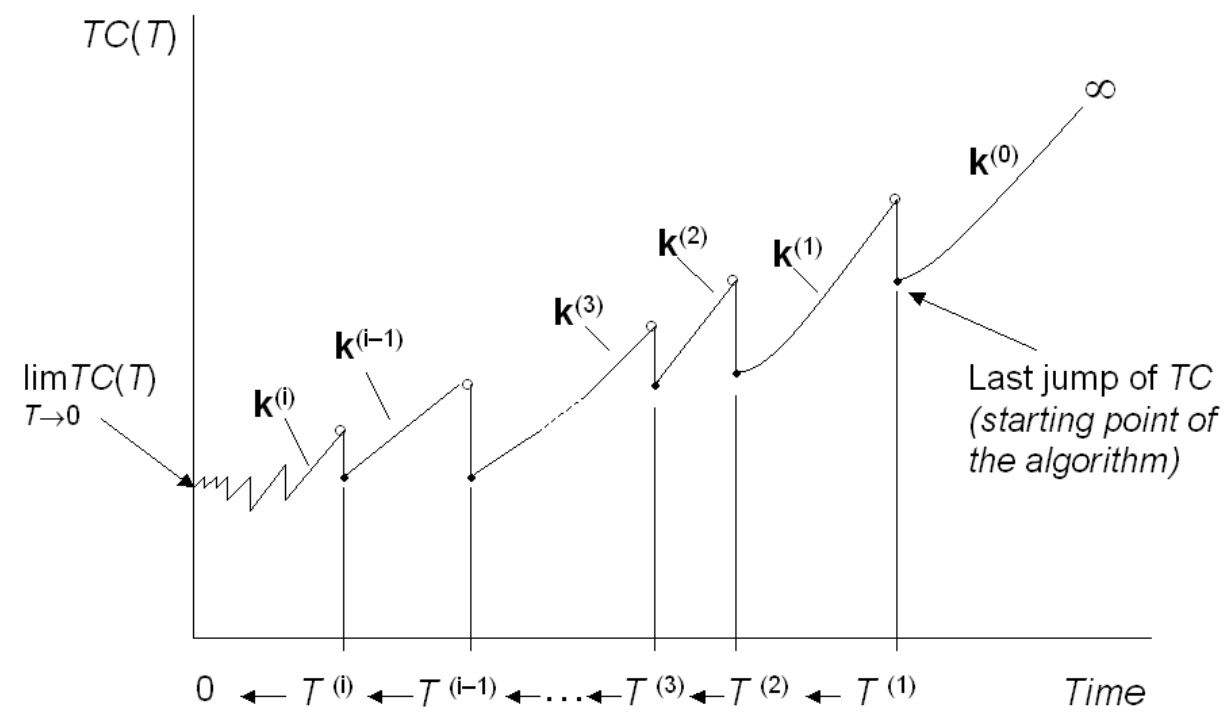

Figure 2. Graphical representation of the global optimisation algorithm

Next we give the formulation of the optimisation algorithm to solve the JRP with MOQ.

\subsection{Global optimisation algorithm for TC}

Step 0. Evaluate the bounds $T_{\text {low }}^{0}$ and $T_{u p p}^{0}$. Determine $\mathbf{k}^{(0)}$ by:

$k_{j}^{(0)}=\left\lceil\frac{M O Q_{j}}{D_{j} T_{u p p}^{0}}\right\rceil$ for $j=1, \ldots, M$, and set $T^{(0)}=\min _{j}\left\{T_{j}^{(0)}\right\}$ where

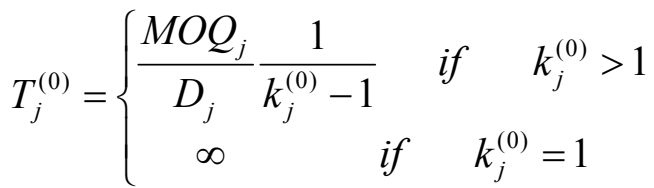

Set $T C_{\min }^{(0)}=\infty$.

Set $n=1$.

Step 1. For $\mathbf{k}^{(n-1)}$ determine $T^{(n)}$ from:

$T^{(n)}=\max _{j}\left\{\frac{M O Q_{j}}{D_{j} k_{j}^{(n-1)}}\right\}$ and set: $J^{(n)}=\left\{j: \max _{j=1, \ldots, M}\left\{\frac{M O Q_{j}}{D_{j} k_{j}^{(n-1)}}\right\}\right\}$ 
Evaluate: $T C\left(T^{(n)}, \mathbf{k}^{(n-1)}\right)$

Evaluate: $T_{n-1}^{*}=\sqrt{\frac{2 A}{\sum_{j} h_{j} k_{j}^{(n-1)} D_{j}}}$

Set: $T C_{\min }^{(n)}=\left\{\begin{array}{lc}\min \left\{T C_{\min }^{(n-1)}, T C\left(T_{n-1}^{*}, \mathbf{k}^{(n-1)}\right)\right\} & \text { if } T_{n-1}^{*} \in\left[T^{(n)}, T^{(n-1)}\right] \\ \min \left\{T C_{\min }^{(n-1)}, T C\left(T^{(n)}, \mathbf{k}^{(n-1)}\right)\right\} & \text { otherwise }\end{array}\right.$

Obtain the elements of the new vector $\mathbf{k}^{(n)}$, according to:

$k_{j}^{(n)}=\left\{\begin{array}{ccc}k_{j}^{(n-1)}+1 & \text { for } & j \in J^{(n)} \\ k_{j}^{(n-1)} & \text { for } & j \notin J^{(n)}\end{array}\right.$

Step 2. If $T^{(n)} \leq T_{\text {low }}$ STOP with $T C_{\min }(T, \mathbf{k})=T C_{\min }^{(n)}$.

Otherwise set $n=n+1$ and GOTO step 1 .

END of the algorithm.

\subsection{Real case}

We apply the global optimisation algorithm to our original data. First we evaluate the lower and upper bounds and the initial vector $\mathbf{k}^{(0)}$ :

Step 0 of the algorithm yields:

$$
\begin{aligned}
& T_{\text {unc }}^{*}=0.199 \text { years } \approx 10.3 \text { weeks } \\
& T_{\text {low }}^{0}=0.0544 \text { years } \approx 3 \text { weeks } \\
& T_{\text {upp }}^{0}=0.728 \text { years } \approx 38 \text { weeks } \\
& \mathbf{k}^{(0)}=\{1,1,1,2,1,2,1,1\}
\end{aligned}
$$

We implemented the above procedure in Maple, searching for the minimal TC inside $\left[T_{\text {low }}^{0}, T_{\text {upp }}^{0}\right]$. For $T_{\text {low }}^{0}=0.0544$ years we get $\mathbf{k}\left(T_{\text {low }}^{0}\right)=\{11,10,11,19,9,19,8,8\}$, and since the changes of the $k_{j}$ 's occur in step sizes of 1 , we can compute the maximum number of steps of the algorithm as follows:

$$
\text { Maximum \# of steps }=\sum_{i: M O Q_{i} / D_{i} \neq M O Q_{j} / D_{j}} k_{k}\left(T_{\text {lo }}^{0}\right)-k_{i}^{(0)}=61 \text { steps }
$$

Note that the maximum number of steps of the algorithm is linear in the number of products. We found the minimum of $T C$ in $T=31$ weeks and $\mathbf{k}=\{1,1,1,2,1,2,1,1\}$. The associated TC is 17840 euros, which represents an improvement of $7.38 \%$ over the iterative algorithm. The improvement is only $0.79 \%$ if we compare it with the total cost with correction factor. 
If we consider the function $T C^{(\mathrm{c})}(T, \mathbf{k})$, the analysis for the bounds on $T$ presented earlier is not valid anymore, since the lower envelope for the function $T C^{(\mathrm{c})}(T, \mathbf{k})$ cannot be obtained by setting $k_{j}=1$ for all $j$. Moreover, the function $T C^{(\mathrm{c})}(T, \mathbf{k})$ does not go to infinity as $T \rightarrow 0$ as we can see in the plot of the graph presented in figure 3 , where $T C^{(\mathrm{c})}(T)$ is defined in an analogous way as $T C(T)$. This behaviour of $T C^{(\mathrm{c})}$ arises from the fact that the term $\Delta(\mathbf{k}) / T$ does not go to infinity as $T$ goes to zero. This requires us to set the lower bound of $T$ in zero, which is not good from a practical point of view.

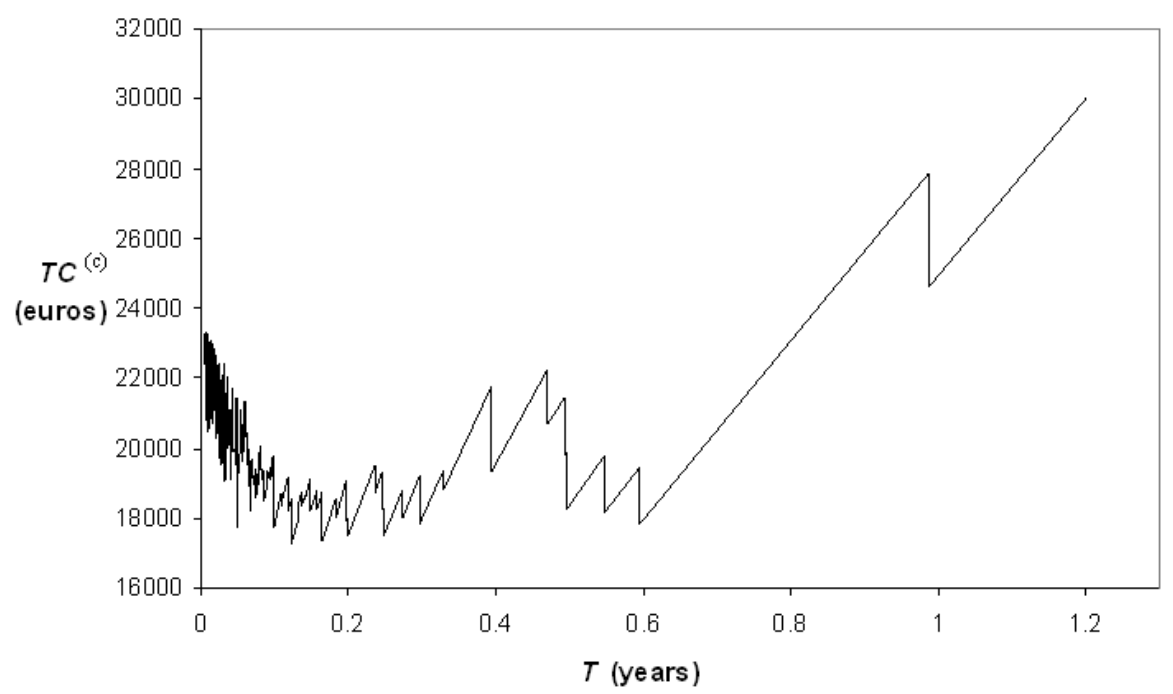

Figure 3. Plot of $T C^{(c)}(T)$

We start our analysis for small $T$. From proposition 1 we have:

$\sum_{i} \frac{1}{T \cdot k_{i}(T)}-\sum_{i \neq j} \frac{1}{T \cdot l c m\left(k_{i}(T), k_{j}(T)\right)} \leq \frac{\Delta(\mathbf{k})}{T} \leq \sum_{i} \frac{1}{T \cdot k_{i}(T)}$

In order to evaluate the limits for $T \rightarrow 0$ in the above inequality first note that:

$k_{j}(T)=\left\lceil\frac{M O Q_{j}}{D_{j} T}\right\rceil \Rightarrow \frac{M O Q_{j}}{D_{j} T} \leq k_{j}(T) \leq \frac{M O Q_{j}}{D_{j} T}+1$

Hence, $\lim _{T \rightarrow 0} T \cdot k_{j}(T)=\frac{M O Q_{j}}{D_{j}}$

To proceed with our analysis we introduce the following notation:

Let $a_{j} \equiv \lim _{T \rightarrow 0} T \cdot k_{j}(T)=\frac{M O Q_{j}}{D_{j}} \quad$ for $\forall j$ 
In the following analysis, we will see that the behaviour of the second term in the LHS of (7) as $T \rightarrow 0$ is very much determined by the nature of the ratios $a_{j} / a_{i}$. Although for practical purposes these ratios can be considered as rational numbers, we found an interesting behaviour of the product $T \cdot \operatorname{lcm}\left(k_{i}(T), k_{j}(T)\right)$ for $T \rightarrow 0$ when the ratios are regarded as irrational numbers, as is the case when demands are continuous variables, rather than discrete. Accordingly, we consider both cases in our analysis. Let $\mathfrak{R} \backslash Q$ denote the set of irrational numbers, where $\mathfrak{R}$ is the set of real numbers and $Q$ the set of rational numbers.

Lemma 1. If $a_{j} / a_{i} \in \mathfrak{R} \backslash Q$, then

$$
\lim _{T \rightarrow 0} \frac{1}{T \cdot \operatorname{lcm}\left(k_{i}(T), k_{j}(T)\right)}=0 .
$$

Proof. Let $\operatorname{lcm}\left(k_{i}(T), k_{j}(T)\right)=n(T) k_{i}(T)=m(T) k_{j}(T)$ for some integers $n(T), m(T)$. Suppose that there is a bounded subsequence $n\left(T^{(r)}\right), m\left(T^{(r)}\right), r=1,2, \ldots$, such that $T^{(r)} \downarrow 0$ as $r \rightarrow \infty$ and $n\left(T^{(r)}\right) \leq K, m\left(T^{(r)}\right) \leq K$ for some $K>0$. Since this implies that there are only finitely many different values of $n\left(T^{(r)}\right), m\left(T^{(r)}\right)$, there exists a second subsequence $n\left(T^{(s)}\right), m\left(T^{(s)}\right)$ of integers such that $n\left(T^{(s)}\right)=n, m\left(T^{(s)}\right)=m \in \mathbb{N}$ and

$$
\frac{n}{m}=\lim _{s \rightarrow \infty} \frac{n\left(T^{(s)}\right)}{m\left(T^{(s)}\right)}=\lim _{s \rightarrow \infty} \frac{k_{j}\left(T^{(s)}\right)}{k_{i}\left(T^{(s)}\right)}=\lim _{s \rightarrow \infty} \frac{T^{(s)} k_{j}\left(T^{(s)}\right)}{T^{(s)} k_{i}\left(T^{(s)}\right)}=\frac{a_{j}}{a_{i}}
$$

However, we assumed that $a_{j} / a_{i}$ was irrational, so there can be no bounded subsequence, hence $n(T), m(T) \rightarrow \infty$ as $T \rightarrow 0$ and

$$
\lim _{T \rightarrow 0} \frac{1}{T \cdot \operatorname{lcm}\left(k_{i}(T), k_{j}(T)\right)}=\lim _{T \rightarrow 0} \frac{1}{\left(k_{i}(T) T\right) \cdot n(T)}=0 \text { as required. }
$$

Remark 1. Lemma 1 implies that the first joint replenishment time of items $i$ and $j$ goes to infinity as $T \rightarrow 0$ if $a_{j} / a_{i}$ is irrational.

Theorem 1. Given $M$ products with demands $D_{1}, \ldots, D_{M}$ and minimum order quantities $M O Q_{1}, \ldots, M O Q_{M}$, if $a_{j} / a_{i} \in \mathfrak{R} \backslash Q \forall i, j$, then

$$
\lim _{T \rightarrow 0} \frac{\Delta(\mathbf{k})}{T}=\sum_{j=1}^{M} \frac{D_{j}}{M O Q_{j}} . \text { Moreover, } \lim _{T \rightarrow 0} T C^{(c)}(T, \mathbf{k})=A \sum_{j=1}^{M} \frac{D_{j}}{M O Q_{j}}+\frac{1}{2} \sum_{j=1}^{M} h_{j} M O Q_{j}
$$

Proof. By lemma 1 we can take limits on both sides of inequality (7) and since both limits exist, the limit of $\Delta(\mathbf{k}) / T$ exists and is equal to the stated value.

Corollary 1. Given two products with $a_{j} / a_{i} \in \mathfrak{R} \backslash Q$, then for every time value $T_{v}$, there is a cycle time $T_{\text {lim }}^{(i, j)}$ for which: 


$$
T_{\text {harm }}^{(i, j)} \equiv T \cdot \operatorname{lcm}\left(k_{i}(T), k_{j}(T)\right)>T_{v} \quad \forall T<T_{\text {lim }}^{(i, j)},
$$

where $T_{h a r m}^{(i, j)}$ denotes the harmonization time of products $i, j$.

Proof. According to lemma 1 , if the condition for the ratios $a_{j} / a_{i}$ is satisfied, $T_{h a r m}^{(i, j)}$ goes to infinity as $T$ goes to zero. Therefore we can find a $T$ close enough to zero for which $T_{\text {harm }}^{(i, j)}>T_{v}$ for any $T_{v}$ large.

The result of corollary 1 can be used to set a practical lower bound on $T$ for the function $T C^{(\mathrm{c})}$.

Although the previous analysis gives us important insight into the behavior of the function $T C^{(\mathrm{c})}$ as $T$ goes to zero, the ratios $a_{j} / a_{i}$ do not need to be irrational numbers, since the demands $D_{j}$ may be truncated to rational numbers. It will come out of our analysis that in this case the function $T C^{(\mathfrak{c})}$ exhibits fluctuations for $T$ close to zero rather than going to a limit as we determined before. The reason behind is the fact that for some $T_{\lim }^{(i, j)}$ arbitrarily small and given $a_{j} / a_{i}$ rational numbers, it is often possible to coordinate products $i$ and $j$ for some $T<T_{\lim }^{(i, j)}$, and the harmonization time $T_{\text {harm }}^{(i, j)}$ does not go to infinity as in the case of $a_{j} / a_{i}$ irrational. Consequently the value of $\Delta(\mathbf{k}) / T$ does not go to a limit as $T \rightarrow 0$ but fluctuates within certain limits. Note however that the upper bound of $\Delta(\mathbf{k}) / T$ as $T \rightarrow 0$ is independent of the nature of $a_{j} / a_{i}$, therefore the previous derivation for the upper bound of $\Delta(\mathbf{k}) / T$ remains valid and we can guarantee that the function $T C^{(\mathrm{c})}$ is bounded for $T \rightarrow 0$.

For the evaluation of a lower bound of $\Delta(\mathbf{k}) / T$ when $T \rightarrow 0$ for $a_{j} / a_{i}$ rational, we will first derive an upper bound on the second term of the LHS of (7), i.e., we analyse the terms $\frac{1}{T \cdot l c m\left(k_{i}(T), k_{j}(T)\right)}$ as $T$ goes to zero. Next we derive bounds on $\Delta(\mathbf{k}) / T$.

We first try to construct a subsequence of $T$ going to zero, say $T^{\left(N_{l}\right)}, l=1,2, \ldots$ s.t. $k_{i}\left(T^{\left(N_{l}\right)}\right)=\left\lceil a_{i} / T^{\left(N_{l}\right)}\right\rceil=N_{l} \cdot m$ and $k_{j}\left(T^{\left(N_{l}\right)}\right)=\left\lceil a_{j} / T^{\left(N_{l}\right)}\right\rceil=N_{l} \cdot n$ where $m, n$ are given integers with $\operatorname{gcd}(m, n)=1$ and $N_{l} \in Z^{+}$.

Hence,

$$
\operatorname{lcm}\left(k_{i}\left(T^{\left(N_{l}\right)}\right), k_{j}\left(T^{\left(N_{l}\right)}\right)\right)=N_{l} \cdot m \cdot n
$$

Such a subsequence of $T$ should satisfy the following system for $N_{l}, l=1,2, \ldots$

$$
\begin{aligned}
& N_{l} m-1<\frac{a_{i}}{T^{\left(N_{l}\right)}} \leq N_{l} m \Rightarrow \frac{a_{i}}{N_{l} m} \leq T^{\left(N_{l}\right)}<\frac{a_{i}}{N_{l} m-1} \\
& N_{l} n-1<\frac{a_{j}}{T^{\left(N_{l}\right)}} \leq N_{l} n \Rightarrow \frac{a_{j}}{N_{l} n} \leq T^{\left(N_{l}\right)}<\frac{a_{j}}{N_{l} n-1}
\end{aligned}
$$


From system (8) it follows that we can find such a sequence of $T$ 's if and only if $\frac{a_{i}}{N_{l} m-1} \geq \frac{a_{j}}{N_{l} n}$ and $\frac{a_{j}}{N_{l} n-1} \geq \frac{a_{i}}{N_{l} m} \quad \forall N_{l}$

Letting $N_{l} \rightarrow \infty$ we obtain:

$a_{j} \leq \frac{N_{l} n}{N_{l} m-1} a_{i} \forall N_{l} \Rightarrow a_{j} \leq \frac{n}{m} a_{i}$ and $a_{j} \geq \frac{N_{l} n-1}{N_{l} m} a_{i} \forall N_{l} \Rightarrow a_{j} \geq \frac{n}{m} a_{i}$

Using both results we get $a_{j}=\frac{n}{m} a_{i}$. This implies that the only $m, n$ for which $\left\lceil a_{i} / T^{\left(N_{l}\right)}\right\rceil=N_{l} \cdot m$ and $\left\lceil a_{j} / T^{\left(N_{l}\right)}\right\rceil=N_{l} \cdot n$ have an infinite number of solutions $N_{l}$ is given by $\frac{a_{i}}{a_{j}}=\frac{m}{n}$. Let us call these values $m_{0}, n_{0}$.

Next note that for $T^{(N)}=\frac{a_{i}}{N \cdot m_{0}}=\frac{a_{j}}{N \cdot n_{0}}, N=1,2, \ldots$ we have such a sequence for which:

$\lim _{N_{l} \rightarrow \infty}\left(T^{\left(N_{l}\right)} \cdot \operatorname{lcm}\left(k_{i}\left(T^{\left(N_{l}\right)}\right), k_{j}\left(T^{\left(N_{l}\right)}\right)\right)\right)=\lim _{N_{l} \rightarrow \infty}\left(T^{\left(N_{l}\right)} \cdot N_{l} \cdot m_{0} \cdot n_{0}\right)=a_{i} \cdot n_{0}=a_{j} \cdot m_{0}$

Now consider an arbitrary $m, n$ given with $\operatorname{gcd}(m, n)=1$ and a time $T$ for which $\left\lceil a_{i} / T\right\rceil=N \cdot m$ and $\left\lceil a_{j} / T\right\rceil=N \cdot n$ for some integer $N>0$

Note that $N$ satisfies the following system:

$$
\begin{gathered}
\frac{a_{i}}{T \cdot m} \leq N<\frac{a_{i}+T}{T \cdot m} \\
\frac{a_{j}}{T \cdot n} \leq N<\frac{a_{j}+T}{T \cdot n}
\end{gathered}
$$

System (9) implies that $\frac{a_{i}}{m} \leq \frac{a_{j}+T}{n}$ and $\frac{a_{j}}{n} \leq \frac{a_{i}+T}{m}$, which yields:

$T \geq \max \left\{a_{i} \frac{n}{m}-a_{j}, a_{j} \frac{m}{n}-a_{i}\right\} \equiv T_{m, n}$ 
Note that $T_{m, n}$ is nonzero if $\frac{m}{n} \neq \frac{a_{i}}{a_{j}}$. Let $T_{i, j}^{*}=\min _{\left\{(m, n) \in \mathbb{N}: m<m_{0}, n<n_{0}\right\}}\left\{T_{m, n}\right\}$.

Now consider a $T<T_{i, j}^{*}$ and let w.l.o.g.

$k_{i}(T)=N^{(T)} \cdot m, k_{j}(T)=N^{(T)} \cdot n$ for some $m, n, N^{(T)} \in \mathbb{N}$

with $\operatorname{gcd}(m, n)=1$, and from (9) we have that: $N^{(T)} \geq \frac{1}{T} \max \left\{\frac{a_{i}}{m}, \frac{a_{j}}{n}\right\}$.

Then,

$T \cdot \operatorname{lcm}\left(k_{i}(T), k_{j}(T)\right)=T \cdot N^{(T)} \cdot m \cdot n \geq\left\{\begin{array}{lc}a_{i} \cdot n & \text { if } \frac{a_{i}}{m}>\frac{a_{j}}{n} \\ a_{j} \cdot m & \text { otherwise }\end{array}\right.$

Since $T<T_{i, j}^{*}$ we cannot have by (11) both $m<m_{0}$ and $n<n_{0}$. Hence, either $m \geq m_{0}$ or $n \geq n_{0}$. In both cases $T \cdot N^{(T)} \cdot m \cdot n \geq a_{i} \cdot n_{0}=a_{j} \cdot m_{0}$.

From the previous analysis we have established the following lemma:

Lemma 2. If $\frac{a_{j}}{a_{i}} \in Q$, then the following holds:

$$
0 \leq \frac{1}{T \cdot \operatorname{lcm}\left(k_{i}(T), k_{j}(T)\right)} \leq \frac{1}{a_{i} \cdot n_{0}} \text { for any } T \leq T_{i, j}^{*}
$$

The previous analysis implies that eventually all pairs of integers $(m, n)$ will die out as $T$ goes to zero, except in the case for which $m=m_{0}$ and $n=n_{0}$. In Fig. 4 we can see a graphic representation of this finding, in which we show a plot of the function:

$$
g(T)=\frac{1}{T \cdot \operatorname{lcm}\left(k_{1}(T), k_{2}(T)\right)}
$$

for products 1 and 2 with corresponding values of $a_{1}$ and $a_{2}$.

Lemma 3. If $\frac{a_{j}}{a_{i}} \in Q$ then for any $T<T_{i, j}^{*}$ the following holds:

$$
\sum_{i} \frac{1}{T \cdot k_{i}(T)}-\sum_{i \neq j} \frac{1}{a_{i} \cdot n_{0}} \leq \frac{\Delta(\mathbf{k})}{T} \leq \sum_{i} \frac{1}{T \cdot k_{i}(T)}
$$

Proof. By lemma 2 and equation (7) the result follows. 


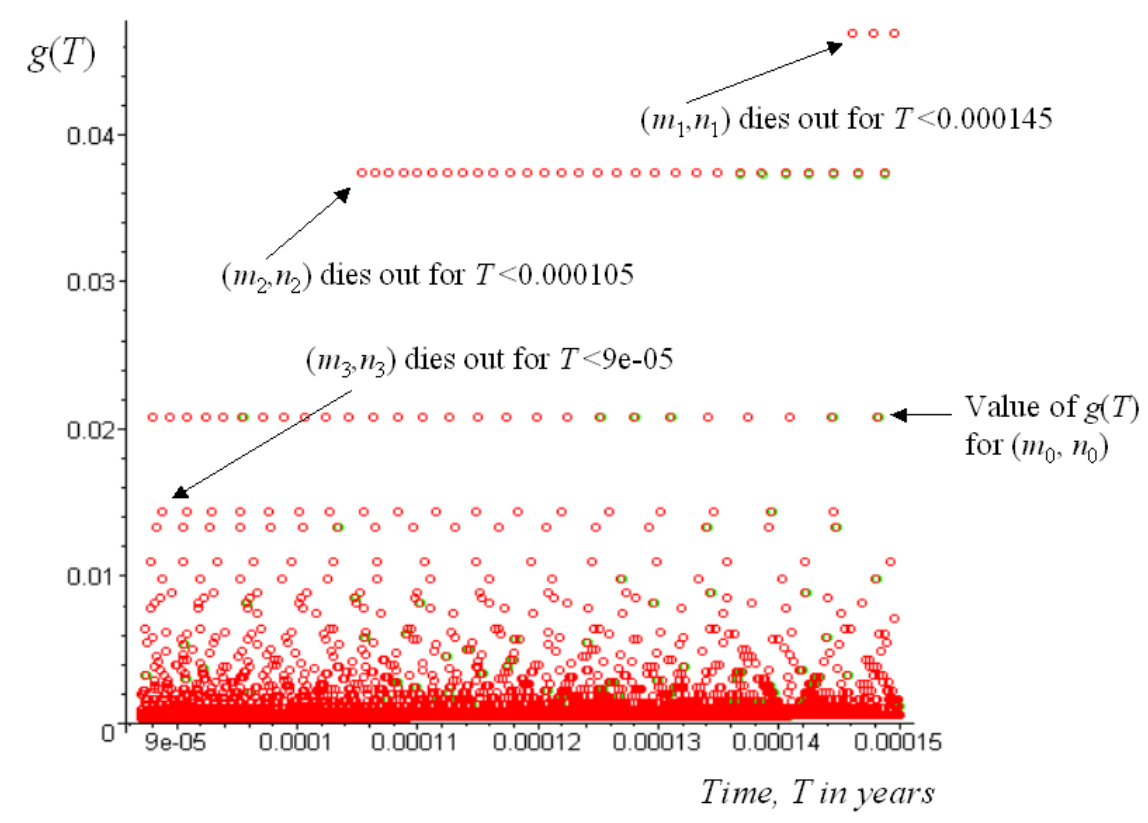

Figure 4. Plot of the function $g(T)$

Lemma 4. If $\frac{a_{j}}{a_{i}} \in Q$, then the following holds:

$\limsup _{T \rightarrow 0} \frac{1}{T \cdot \operatorname{lcm}\left(k_{i}(T), k_{j}(T)\right)}=\frac{1}{a_{i} \cdot n_{0}}$

and

$\liminf _{T \rightarrow 0} \frac{1}{T \cdot \operatorname{lcm}\left(k_{i}(T), k_{j}(T)\right)}=0$

Proof. A large part of this lemma follows from lemma 2 and the analysis preceding it. What remains to be proved is that the liminf as $T \rightarrow 0$ is indeed 0 . For this part suppose w.1.o.g. that $a_{i}>a_{j}$, implying that $k_{i}(T)>k_{j}(T)$ for $T$ small. Note that as $T \rightarrow 0, k_{i}(T)$ takes all possible integers $1,2, \ldots$ Let $\alpha_{1}, \alpha_{2}, \alpha_{3}, \ldots$ be an increasing sequence of prime numbers and let $T^{\left(\alpha_{l}\right)}$ be the $T$-values for which $k_{i}\left(T^{\left(\alpha_{l}\right)}\right)=\alpha_{l}, l=1,2, \ldots$ Now note that for $T$ small enough

$\operatorname{lcm}\left(k_{i}\left(T^{\left(\alpha_{l}\right)}\right), k_{j}\left(T^{\left(\alpha_{l}\right)}\right)\right)=k_{i}\left(T^{\left(\alpha_{l}\right)}\right) \cdot k_{j}\left(T^{\left(\alpha_{l}\right)}\right)$ and hence,

$\lim _{T^{\left(\alpha_{l}\right)} \rightarrow 0} \frac{1}{T^{\left(\alpha_{l}\right)} \cdot \operatorname{lcm}\left(k_{i}\left(T^{\left(\alpha_{l}\right)}\right), k_{j}\left(T^{\left(\alpha_{l}\right)}\right)\right)}=\lim _{T^{\left(\alpha_{l}\right)} \rightarrow 0} \frac{1}{T^{\left(\alpha_{l}\right)} \cdot k_{i}\left(T^{\left(\alpha_{l}\right)}\right) \cdot k_{j}\left(T^{\left(\alpha_{l}\right)}\right)}$

$=\frac{1}{\lim _{T^{\left(\alpha_{l}\right)} \rightarrow 0}\left(T^{\left(\alpha_{l}\right)} \cdot k_{i}\left(T^{\left(\alpha_{l}\right)}\right)\right) \cdot \lim _{T^{\left(\alpha_{l}\right)} \rightarrow 0}\left(k_{j}\left(T^{\left(\alpha_{l}\right)}\right)\right)}=\frac{1}{a_{i} \cdot \lim _{T^{\left(\alpha_{l}\right)} \rightarrow 0}\left\lceil\frac{a_{j}}{T^{\left(\alpha_{l}\right)}}\right\rceil}=0$. 
Theorem 2. Given $M$ products with demands $D_{1}, \ldots, D_{M}$ and minimum order quantities $M O Q_{1}, \ldots, M O Q_{M}$, if $a_{j} / a_{i} \in Q \forall i, j$ then

$$
\liminf _{T \rightarrow 0} T C^{(c)}(T, \mathbf{k})=A\left(\sum_{i: a_{i} \neq a_{j}} \frac{D_{i}}{M O Q_{i}}-\sum_{i: a_{i} \neq a_{j}} \frac{1}{\left.a_{i} \cdot n_{0}\right)}\right)+\frac{1}{2} \sum_{j=1}^{M} h_{j} M O Q_{j}
$$

and

$$
\underset{T \rightarrow 0}{\limsup } T C^{(c)}(T, \mathbf{k})=A \sum_{j=1}^{M} \frac{D_{j}}{M O Q_{j}}+\frac{1}{2} \sum_{j=1}^{M} h_{j} M O Q_{j}
$$

Proof. By lemma 4 we can evaluate the limit as $T \rightarrow 0$ on the LHS of inequality (7) and since the limit exists, the first part of the theorem follows. For the second part take the limit on the RHS of (7), and since this limit exists and it is independent of $a_{j} / a_{i} \forall(i, j)$ the claim of the theorem follows.

Remark 2. If $a_{j} / a_{i}=1$ then the products can be combined and considered as one product, so we do not need to invoke theorem 2, but the result of theorem 1 holds.

By lemma 2 and the analysis preceding it, we can evaluate a lower bound on $T$ for the function $T C^{(\mathrm{c})}$ as follows:

$$
T_{\text {low }}^{(c)}=\min _{(i, j): a_{i} \neq a_{j}}\left\{T_{i, j}^{*}\right\}
$$

Remark 3. The above procedure to evaluate a lower bound on $T$ can yield very small values, which may not be useful for practical purposes. In such a case, a practical lower bound on the review time $T$ should be established, say one day or one hour, which is a reasonable assumption for most inventory tracking systems.

An upper bound on $T$ for the function $T C^{(\mathrm{c})}$ can be evaluated as follows:

Since $k_{j}(T)=\left\lceil\frac{M O Q_{j}}{D_{j} T}\right\rceil$, there is a time $T^{(1)}$ such that for any $T \geq T^{(1)}, k_{j}=1$ for all $j$, given by:

$$
T^{(1)}=\max _{j}\left\{\frac{M O Q_{j}}{D_{j}}\right\}
$$

Since $\Delta(\mathbf{k})=1$ if any of the $k_{j}=1$ we have:

$$
T C^{(c)}(T, \mathbf{k})=\frac{A}{T}+\frac{1}{2} T \sum_{j=1}^{m} h_{j} D_{j} \text { for } T \geq T^{(1)}
$$


Since the last function is concave with a minimum value in $T_{u n c}^{*}$, it follows that if $T_{u n c}^{*} \geq T^{(1)}$ the function $T C^{(\mathrm{c})}$ will monotonically increase for $T \geq T_{u n c}^{*}$, otherwise it will monotonically increase for $T \geq T^{(1)}$. Therefore the upper bound on $T$ for the function $T C^{(c)}$ is given by:

$$
T_{u p p}^{(c)}=\max \left\{T_{u n c}^{*}, T^{(1)}\right\}
$$

Note that the previous upper bound for $T$ is generally larger than the one found when considering $T C$ without correction factor.

Now that we have defined lower and upper bounds on $T$ for $T C^{(\mathrm{c})}$, we can formulate a global optimisation algorithm to find the minimum of the function. We will do this based on the previous algorithm for $T C$ without correction factor with the modified lower and upper bounds on $T$.

\subsection{Global optimisation algorithm for $T C^{(c)}$}

Step 0. Evaluation of lower and upper bounds on $T$. Set $k^{(0)}=\{1,1, \ldots, 1\}$ and select:

$$
T^{(1)}=\max _{j}\left\{\frac{M O Q_{j}}{D_{j}}\right\}
$$

Evaluate $T_{u n c}^{*}$ and set $T_{u p p}^{(c)}=\max \left\{T_{u n c}^{*}, T^{(1)}\right\}$.

Set $T_{\text {low }}^{(c)}=\min _{(i, j): a_{i} \neq a_{j}}\left\{T_{i, j}^{*}\right\}$, or use a practical lower bound.

Step $1(n \geq 1)$ and Step 2. Replace the function $T C$ by $T C^{(\mathrm{c})}$ in the global optimisation algorithm for $T C$ and use the following formula for the evaluation of $T^{*}$ :

$$
T_{n-1}^{*}=\sqrt{\frac{2 A \Delta\left(\mathbf{k}^{(n-1)}\right)}{\sum_{j} h_{j} k_{j}^{(n-1)} D_{j}}}
$$

Applying the global optimisation procedure to $T C^{(\mathrm{c})}(T, \mathbf{k})$ we obtained the following results:

$T_{\text {upp }}=0.986$ years $\approx 51$ weeks

$T_{\text {low }}=3.21 \times 10^{-6}$ year $\approx 0.03 \mathrm{hrs}$. (we ran the algorithm using a practical lower bound $T_{\text {low, } \text { pract }}=0.0001$ year $\approx 1$ hour $)$.

$\mathbf{k}\left(T_{\text {low,pract }}\right)=\{5464,4957,5954,9862,4714,9862,3933,3933\}$

$T C_{\min }=17297$ euros $=3056$ (ordering costs) +14241 (inventory costs)

$T_{\text {opt }}=6.44$ weeks $\approx 45$ days

$\mathbf{k}_{\text {opt }}=\{5,4,5,8,4,8,4,4\}$

Using theorem 2 we evaluated the following bounds for the function $T C^{(\mathrm{c})}$ as $T \rightarrow 0$ : 


$$
\begin{aligned}
& \liminf _{T \rightarrow 0} T C^{(c)}(T, \mathbf{k})=23354 \text { euros } \\
& \limsup _{T \rightarrow 0} T C^{(c)}(T, \mathbf{k})=23645 \text { euros }
\end{aligned}
$$

As before, the maximum number of steps of the algorithm is given by:

$$
\text { Maximum \# of steps }=\sum_{i: M O Q_{i} / D_{i} \neq M O Q_{j} / D_{j}} k_{i}\left(T_{\text {pract }}\right)-k_{i}^{(0)}=34878 \text { steps }
$$

As for the algorithm for $T C$ the number of steps of the algorithm for $T C^{(\mathrm{c})}$ is linear in the number of items, since it is equal to the sum of jumps of the function $T C^{(\mathrm{c})}$ between $T_{\text {low }}$ and $T_{\text {upp }}$.

The solution we found for the optimisation of $T C^{(\mathrm{c})}$ is quite different from the one of $T C$ for the same data. Although the improvement in the objective value is only $3 \%$, the solution using the correction factor gives a much smaller value of $T_{\text {opt }}$, with the consequently use of higher values for the frequencies $k_{j}$. In this case the times for replenishments of the products given by $k_{j} T$ do not differ much from the previous solution of $T C$, but the opportunities per year to review the system are higher when using the correction factor. More specifically note that the average time of ordering in the previous solution is some 90 days, whereas this figure in the solution for $T C$ without correction factor is 217 days.

The disadvantage of the above algorithm compared with the one for $T C$, is that in order to guarantee the quality of the solution and since the function $T C^{(\mathrm{c})}$ is much more sensitive to $T$, the number of steps in the latter is considerably higher.

\section{Conclusions}

In this paper we showed that it is possible to solve the JRP with minimum order quantities by applying a global optimisation procedure. We derived proper lower and upper bounds for the basic cycle time $T$ using both formulations of the JRP with constraint, with and without correction factor, and we proposed a new solution method based on the formulation of the problem given by Wildeman et al [7]. We demonstrated that an iterative procedure approach to find simultaneously the optimal values for the $k_{j}$ 's and $T$, as the one used by Viswanathan [6] to improve the bounds, does not perform well in this case.

\section{Acknowledgments}

The authors are grateful to Prof. Dr. Sotirios Papachristos for valuable comments on the paper. 


\section{References}

[1] J.S. Dagpunar, Formulation of a multi item single supplier inventory problem, Journal of the Operational Research Society 33 (1982) 285-286.

[2] M.J.G. van Eijs, A note on the joint replenishment problem under constant demand, Journal of the Operational Research Society 44 (1993) 185-191.

[3] R.Y.K. Fung, X. Ma, A new method for joint replenishment problems, Journal of the Operational Research Society 52 (2001) 358-362.

[4] S.K. Goyal, Determination of optimum packaging frequency of items jointly replenished, Management Science 21 (1974) 436-443.

[5] E. Porras, R. Dekker, Controlling inventories in a supply chain, in: Discussion papers of the Tinbergen Institute, TI 2003 (012/4) (2003).

[6] S. Viswanathan, A new optimal algorithm for the joint replenishment problem, Journal of the Operational Research Society 47 (1996) 936-944.

[7] R.E. Wildeman, J.B.G. Frenk, R. Dekker, An efficient optimal solution method for the joint replenishment problem, European Journal of Operational Research 99 (1997) 433-444. 